\title{
UN MODELO DE MONITOREO DE LOS APRENDIZAJES DURANTE LA FORMACIÓN INICIAL DOCENTE: UNA OPORTUNIDAD DE CONTAR CON EVIDENCIA CONCRETA DE LA CALIDAD DE LOS PROGRAMAS
}

\section{Introducción}

La calidad de la educación se juega en parte importante en la calidad de los profesores (Stuhlman y Pianta, 2009), la que a su vez depende en gran medida de la calidad de los programas de formación inicial (Darling-Hammond, 2006 y 2012; Levine, 2006).

Por esto, uno de los desafíos centrales para mejorar la calidad de la educación que nuestro país ofrece a sus niños es perfeccionar la formación de los docentes que la entregarán. El logro de este desafío ha llevado a nuestro país a importantes avances, como la creación de estándares de formación inicial docente por parte del Ministerio de Educación, las becas dirigidas a reclutar estudiantes con mejor puntaje PSU, la entrega de importantes recursos por parte del Estado a las universidades formadoras de profesores que se comprometan con avances notables, la prueba INICIA y la obligatoriedad de la acreditación para las carreras de Pedagogía.

El presente artículo tiene como fin mostrar el resultado de los esfuerzos de la Facultad de Educación de la Universidad Diego Portales (UDP en adelante) por contar con evidencia acerca de los logros de aprendizaje de sus estudiantes, mediante la creación de un sistema que mide los aprendizajes adquiridos por los estudiantes de Pedagogía. Se relatará el proceso con el que se construyó este sistema de monitoreo, el modo cómo se recolectó evidencia de los aprendizajes y el uso que se espera dar a la información obtenida.

Asimismo, se argumentará que este tipo de evidencia es de gran valor y utilidad, tanto para juzgar la calidad de los programas de Pedagogía como para que cada entidad educacional automonitoree 
sus logros y debilidades a tiempo, asegurando así la formación de los mejores profesores.

\section{1. ¿Cuál es la información más relevante para juzgar los programas de formación inicial?}

La literatura identifica entre los factores estratégicos en el aseguramiento de la calidad durante la preparación de los profesores (Barber y Mourshed, 2008; Wang, Coleman, Coley y Phelps, 2003) los siguientes:

- Reclutar a quienes estudian para ser profesores de entre los mejores postulantes.

- Monitorear y acreditar la calidad de los programas de formación de profesores y sus resultados de aprendizaje además de sus procesos.

- Establecer requisitos para entrar al ejercicio profesional u obtener una certificación definitiva como profesor.

Las nuevas iniciativas del Ministerio de Educación, en efecto, se han focalizado en las recomendaciones respecto del ingreso y egreso de los programas de formación, a saber, en el esfuerzo de reclutar a los mejores estudiantes (becas) y en medir los conocimientos de salida (prueba INICIA). Así, la prueba INICIA se dirige específicamente a medir el logro de aprendizajes durante el trayecto de los programas de FID, pero es una medición que al realizarse ya finalizada la formación docente, no permite un espacio para medidas remediales que puedan llenar vacíos relevantes en una trayectoria académica. En contraste, los sistemas más sólidos para asegurar la calidad de los egresados de programas de Pedagogía se basan en demostrar resultados, es decir, en exponer evidencias de las competencias y conocimientos que están adquiriendo durante su formación quienes se educan para ser futuros docentes (Ingvarson, Schwille, Rowley, Tatto, Senk, Peck, 2006 y 2011; Rothstein, Jacobsen y Wilder, 2009).

Así, contar con evidencia durante la trayectoria de la formación inicial docente (FID) permite contar con la oportunidad de asegurar que la mayor parte de los profesores en formación, efectivamente, cuente con las competencias mínimas para ser un buen docente en el futuro. 


\section{Sistema de monitoreo y evaluación de los aprendizajes desarrollado por la Facultad de Educación de la UDP}

Frente al escenario descrito, la Facultad de Educación de la UDP se propuso crear un sistema de monitoreo y evaluación que permitiera contar con datos oportunos, en distintos puntos del proceso de formación, acerca de los aprendizajes de sus estudiantes.

El propósito del proyecto fue justamente contar con evidencia que reflejara los avances de los alumnos, con un especial acento en recoger información durante todo su trayecto académico, lo que, a su vez, permitiera realizar ajustes oportunos para asegurar procesos de aprendizaje eficientes para la mayor parte de los futuros docentes. De este modo, los datos se pensaron como elementos que sirvieran de insumos para la mejora de los programas de los cursos, la malla académica y de las prácticas de enseñanza. Asimismo, se esperaba que esta evidencia sirviera como un indicador palpable de la calidad del programa de FID ofrecido por la Facultad.

El denominado Sistema de Monitoreo y Evaluación de los Aprendizajes (SME) se diseñó durante 2010, con el apoyo de un proyecto Mecesup. Para el logro de este desafío fue necesario un trabajo participativo de los actores más relevantes de la Facultad: directores/as, académicos/as y estudiantes. Asimismo, fue crucial conocer experiencias del extranjero y contar con expertos para la asesoría. Por otra parte, se debieron tomar importantes decisiones durante el proceso, acerca de qué medir, con qué información contar en una primera etapa, cómo medir los aprendizajes, qué hacer con la información, etc. Cada una de estas decisiones fue moldeando el sistema.

A continuación, el presente escrito describirá en mayor detalle cada una de las cuestiones antes mencionadas, como también el proceso de creación del SME.

\subsection{Necesario trabajo en equipo y consejo de expertos}

Como primera medida para enfrentar el desafío de crear un sistema de recogida de datos que abarcaría la labor de muchos de los actores de la Facultad, se conformó un amplio equipo de trabajo y se aseguró 
la participación de la mayor cantidad de académicos. Esto resultó fundamental, pues incidiría positivamente en el grado de aceptación del sistema y disminuiría la resistencia de los profesores a contribuir con datos provenientes de sus cursos y evaluaciones.

Asimismo, se visitaron experiencias extranjeras en las cuales se recogiera evidencia de aprendizajes en Facultades de Educación de universidades de prestigio, como el Wheelock College y la University of Wyoming en Estados Unidos (ambas con acreditación nacional en formación docente). Se contó además con expertos internacionales quienes apoyaron el proceso de trabajo y viajaron a Chile para asesorarlo.

A lo largo del proceso de creación del SME se debieron tomar muchas decisiones, las que se conversaban en un equipo consultivo compuesto por el decano, directoras y académicos. Los modelos propuestos luego fueron socializados en reuniones ampliadas de académicos y profesores y también de estudiantes, para conocer sus opiniones y comentarios.

\section{2. ¿Qué aprendizajes observar?}

La primera pregunta a la que este esfuerzo se enfrentó fue qué aprendizajes debemos tomar en cuenta como evidencia relevante de los procesos de aprendizaje en la FID. La respuesta a esta cuestión era clave, pues, a su vez, aclararía qué cursos pasarían a ser la fuente de los datos que alimentarían el sistema.

Para el año en que este diseño se trabajó (2010), la respuesta más acertada fue levantar datos que correspondieran a las competencias y aprendizajes que el perfil de egreso de las carreras de Pedagogía declaraba, como también hacer una búsqueda en la literatura respecto de las habilidades centrales de un buen profesor. $^{7}$

Una revisión de la teoría y de los estudios referidos a los conocimientos relevantes para la profesión docente (Shulman, 1987;

7 Otra fuente relevante de información respecto de este asunto pudo haber sido la de los estándares de FID del Mineduc, que fueron recién publicados en 2011. 
Darling Hammond y Bransford, 2005; Ben-Peretz, 2011; Oliva, Díaz, Larrosa, Contreras y Miranda, 2010; Berliner, 1986; Ávalos, Cavada, Pardo y Sotomayor, 2010) permitió derivar distintos ámbitos del saber que debe poseer un profesor. Si bien en la literatura se han propuesto distintas clasificaciones, existe bastante consenso respecto de la necesidad de conocimientos acerca de los contenidos por enseñar (o saber disciplinario), en torno a enseñar y aprender (o de didáctica general), del currículo, de los contextos de la enseñanza y el llamado saber pedagógico del contenido (Shulman, 1987) (o didáctica específica). Este último constructo involucra, por una parte, un conocimiento específico del estudiante que aprende un determinado contenido o habilidad y, por otra, la capacidad de transformar el conocimiento disciplinario para hacerlo susceptible de ser aprendido por el o los estudiantes en cuestión.

Sin embargo, otros autores proponen que solo contar con conocimientos pedagógicos y disciplinarios, también llamados conocimientos académicos, no es suficiente para hacer a un buen profesor. Además, se propone como necesario el manejo de habilidades básicas a nivel de lenguaje y de la abstracción matemática, teniendo presente que el desarrollo de las mismas son consideradas fundamentales para el ejercicio de las competencias pedagógicas (Velásquez, Peronard, Alonzo, Ibáñez y Órdenes, 2006).

Respecto del dominio de la lengua escrita que deberían poseer los estudiantes, es un hecho que la decisión de qué y cómo enseñar en las universidades chilenas hoy debe involucrar una consideración de las debilidades en lectura y escritura de los estudiantes universitarios denominados de primera generación (Latorre, Aravena, Milos, García, 2010; Ruffinelli y Guerrero, 2009). Este problema se ha documentado en Chile respecto de las llamadas habilidades básicas que deben poseer los estudiantes de carreras de educación (Larrondo, Lara, Figueroa, Rojas, Caro, 2007) y se expresa en la actualidad en la existencia creciente de cursos remediales y propedéuticos puestos en funcionamiento en las distintas universidades. Se trata de un fenómeno que parece transversal a las diferentes instituciones y que ha dado origen a pruebas de habilidades comunicativas y a fondos 
públicos que buscan frenar la deserción en los primeros años de formación (Centro de Microdatos, 2008).

A lo anterior, la Comisión Nacional para la Acreditación de la Formación de Profesores de EE.UU. agrega dos ámbitos de dominio (NCATE, 2008):

- La capacidad de reflexionar acerca de sus creencias y expectativas respecto de las características de los niños a los que enseñará y sobre el proceso de enseñanza/aprendizaje en general. Esto, pues para enseñar de manera efectiva a un grupo heterogéneo de niños el profesor debe partir con las más altas expectativas y con una postura que asegure las mismas oportunidades para todos sus estudiantes.

- La capacidad de evaluar constantemente su labor, de manera de contar con información para mejorarla. Los profesores necesitan revisar constantemente lo que sus estudiantes están realmente aprendiendo y así reevaluar sobre la marcha su plan de estudio y estrategias de enseñanza.

Una segunda cuestión fue definir los aprendizajes que el SME miraría a lo largo del currículo. Para esto se contrastó el perfil de egreso de las carreras de Pedagogía de la UDP (Pedagogía Básica y Parvularia) con lo que la literatura señalaba como las principales características de un profesor efectivo. Muchas de las competencias de este perfil efectivamente coincidían con lo revisado en esta materia. De este modo, se trabajó con el conjunto de competencias del perfil que resultara más representativo de lo expuesto por la literatura especializada en formación de profesores.

A partir de este ejercicio, se agruparon los aprendizajes que serían monitoreados en cinco grandes ejes temáticos que el SME se haría cargo de seguir a lo largo de la formación docente. Estos ejes, a su vez, agrupan las competencias del perfil de egreso con las que se trabajaría. Estos son: el eje de habilidades básicas, el de creencias y opiniones sobre la enseñanza y el aprendizaje, el de conocimiento disciplinario y su didáctica en lenguaje, el de indagación y el de aprender a enseñar (ver Figura 1). 
Figura 1: Ejes que constituyen el SME

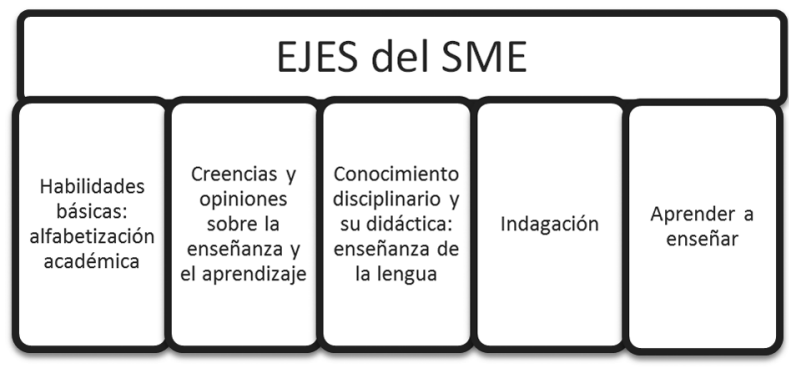

El eje de habilidades básicas agrupa en el SME la evidencia acerca del dominio de la lengua escrita que poseen los estudiantes, tanto las referidas a la lectura como a la escritura académica.

El eje de creencias y opiniones sobre la enseñanza y el aprendizaje levanta evidencia acerca de la capacidad de los futuros profesores de identificar y reflexionar en torno a sus propias creencias y a las expectativas que tienen acerca de los diferentes tipos de alumno a los que en el futuro enseñarán; así como también respecto de las principales creencias asociadas a las diferentes teorías sobre el aprendizaje y la capacidad de diseñar propuestas didácticas que reflejen las altas expectativas puestas en todos los alumnos.

El eje de conocimiento disciplinario y enseñanza de la lengua persigue saber si los estudiantes, a lo largo de su formación docente, han adquirido el conocimiento disciplinario/teórico para la enseñanza de la lectura y escritura y la oralidad de $1^{\circ}$ a $6^{\circ}$ básico, en el caso de la carrera de Educación Básica; y el conocimiento disciplinario/ teórico acerca de la adquisición y desarrollo de la lengua (0-3 años), de la alfabetización emergente y de la literatura infantil, en el caso de Educación Parvularia. Por su parte, el tipo de evaluación incluida en este eje es coherente con aquello que señala la literatura respecto de lo que deben ser capaces de hacer los profesores con su conocimiento, que es usarlo articuladamente para planificar una clase y para fundamentar esa planificación.

El eje de indagación persigue arrojar datos que muestren que, a lo largo de su formación docente, los estudiantes han adquirido 
las competencias para generar una pregunta de investigación y responderla por medio de la literatura; de aplicar lo anterior a indagar sobre temas, áreas o casos que se enfrentan cotidianamente en las escuelas; de generar propuestas de investigación y de ejecutarlas con el fin de mejorar y actualizar de manera continua su labor como profesores.

Por último, el eje de aprender a enseñar levanta datos de las destrezas docentes que revelan los estudiantes cuando están a cargo de un aula real, típicamente durante su práctica profesional, como manejo pedagógico del aula y planificación de clases de calidad.

El diseño del SME contempla, en una primera etapa, los ejes antes mencionados. Estos no abarcan -en ningún caso- todas las disciplinas que un profesor debe manejar. Por ejemplo, los ejes de habilidades básicas y de conocimiento disciplinario solo abarcan datos del área de lenguaje y comunicación. Esto responde al hincapié que los expertos hicieron respecto de instalar un sistema de manera gradual, comenzando con una cantidad manejable de información para, una vez que ya el SME esté instalado, hacerlo más complejo. Así, si bien se considera que son de gran importancia tanto las habilidades básicas de pensamiento en las áreas de lenguaje como las de matemática, la primera versión del SME abarca solo el área de lenguaje, pues se decidió trabajar habilidades generales de alto impacto para un mayor número de asignaturas, como son las destrezas de lenguaje. Coincidentemente con lo anterior, fue también el área de lenguaje la primera que se decidió medir el eje de conocimiento disciplinario y su didáctica. No obstante, en el futuro el SME incorporará paulatinamente las demás áreas disciplinares, en concordancia con los estándares orientadores para las diferentes Pedagogías.

\section{3. ¿Qué cursos participan?}

Una vez definidos los cinco ejes temáticos del SME, se procedió a seleccionar los cursos de las mallas curriculares de Educación Parvularia y Educación Básica que ya estaban asociados a las competencias del perfil elegidas para ser monitoreadas, y que a su vez se asocian a las grandes áreas temáticas de cada eje. De este modo, 
se constituyeron cinco líneas de cursos representativas de los ejes a evaluar.

Debido a que uno de los objetivos del SME era contar con datos que oportunamente informaran acerca de los logros -o problemasen la adquisición de los aprendizajes más centrales para cada uno de los ejes descritos, es que se priorizó obtener evidencia de cursos que cubrieran diferentes momentos de las mallas curriculares.

En honor a la síntesis, no se detallará cada una de las líneas de cursos asociadas a cada eje del SME, sino que se ejemplificará el proceso de construcción con dos de ellas, siempre para la carrera de Educación Básica.

Un eje que ayuda a clarificar la manera en que se levantan los datos del sistema es el de indagación. Este eje persigue recoger información que dé cuenta del logro de las competencias del perfil de egreso:

- Emplea diversas estrategias de investigación para conocer, analizar y reflexionar sobre problemas que existen en la escuela y en el aula.

- Utiliza la autoevaluación y la indagación reflexiva que articula la teoría con la práctica para analizar y mejorar su desempeño.

Como se puede apreciar en el Figura 2, este eje de cursos se compone por asignaturas que se imparten en cuatro tiempos del programa de formación (que tiene en total ocho semestres), con cursos al inicio, al medio y al final del programa.

Figura 2: Cursos que componen el eje de indagación en el programa de Educación Básica

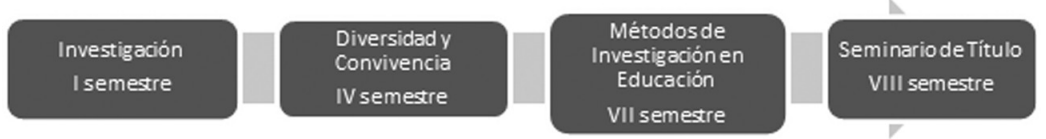

Como ya se ha explicitado previamente, la selección de los cursos se realizó de acuerdo con los que originalmente ya estaban asociados desde la creación de la malla curricular, a cada una de las competencias del perfil de egreso seleccionadas para ser parte del SME. Pero en este eje, además, se agregó uno que no estaba originalmente 
vinculado con las competencias de indagación, que es el curso de Diversidad y Convivencia del IV semestre. Esto último se verificó porque los cursos asociados a las competencias de indagación se concentraban al principio y al final del plan de estudio, lo que no permitía contar datos para este eje en la mitad de la carrera. Esto constituía un problema, porque al no tener datos durante ese periodo, solo se podrían detectar fallas en los aprendizajes de esta área hacia el fin de los estudios, con la consecuente falta de oportunidades de intervención. En el apartado siguiente se explicará en mayor detalle qué tipo de evidencia se levanta en cada curso.

Otro eje especialmente interesante es el de habilidades básicas, que agrupa datos que demuestren que los alumnos de la Facultad están adquiriendo las destrezas de una alfabetización académica. Este eje se asocia directamente con la competencia del perfil de egreso que plantea que el estudiante que finaliza el programa de Educación Básica «se comunica con claridad en forma oral y escrita». Para el trabajo de estos aprendizajes, la Facultad se decidió por una modalidad transversal que interviniera a lo largo de la malla de cursos, lo que se diferencia de un modelo más tradicional donde existen uno o más cursos especiales en los cuales estas habilidades se refuerzan. Este modelo transversal facilita el que los estudiantes adquieran el dominio del lenguaje escrito en cursos regulares de la malla donde se trabaja la escritura y la comunicación oral en ámbitos académicos y en el marco de los aprendizajes relevantes para ser profesor. Esto se traduce en una línea de cursos donde se lleva a cabo una intervención para enseñar a comunicarse a nivel académico. Adicionalmente, este eje contempla mediciones de entrada y salida de habilidades básicas de lenguaje, por una prueba estandarizada, cuyos resultados permiten conocer el rendimiento de cada cohorte a la entrada y a la salida del programa de estudio, siendo posible así comparar su rendimiento. Esto último tiene como fin buscar potenciales ganancias en estas habilidades una vez que han completado su formación. Para este fin se utiliza un test $^{8}$ dirigido a medir habilidades básicas de lenguaje, validado en

8 Cada prueba está constituida por 30 ítems de alternativas que exigen las siguientes destrezas: localizadas (encontrar información explícita); cíclicas (relacionar un conjunto de información); integradas (inferir información, identificar causas y efectos, reconocer y analizar alternativas de solución de problemas) y generativas (producir respuestas propias). 
aplicaciones masivas a estudiantes chilenos de Pedagogía en 2001 y 2002 (Ávalos, 2002; Larrondo, 2001) en el marco del Programa de Fortalecimiento de la Formación Inicial Docente (PFFID), impulsado y coordinado por el Ministerio de Educación.

Se puede apreciar en la Figura 3 que el eje habilidades básicas seleccionó la siguiente línea de cursos, los que cubren el tiempo inicial, medio y final de la carrera.

Figura 3: Cursos que componen el eje de habilidades básicas: alfabetización académica, en el programa de Educación Básica

TEST HB LENGUAJE

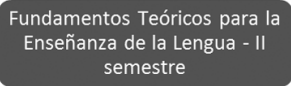

Lenguaje y Comunicación y su Didáctica - IV semestre
TEST HB LENGUAJE

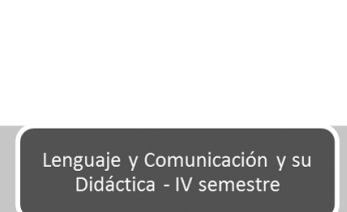

Métodos de Investigación en Educación - VII semestre

Como podrá apreciarse, uno de los cursos -Métodos de Investigación en Educación (VII semestre)- es compartido por ambas líneas de cursos descritas. Esto significa que el mencionado curso tendrá que informar dos tipos de evidencias, una dirigida al eje de indagación y otra al de habilidades básicas: alfabetización académica.

\section{4. ¿Qué información se levanta?}

Ya se ha explicado que cada uno de los cursos seleccionados, en cada uno de los ejes, estaba ya originalmente asociado a cada una de las competencias del perfil de egreso que agrupan los ejes temáticos del SME. La asociación de los cursos a las competencias del perfil de egreso fue parte de una reestructuración de la malla curricular de las carreras de Pedagogía de la Facultad de Educación de la UDP, que se diseñó en 2008 y se hizo efectiva a partir de 2010. La creación de la nueva malla, así como también el diseño del SME, se llevaron a cabo siguiendo las orientaciones del procedimiento del backwards design (de atrás hacia delante), donde primero se define un resultado (el perfil de egreso), para luego definir los cursos necesarios para lograr ese perfil. Para el caso del SME, las competencias del perfil de egreso cuyos logros se decidió monitorear se convierten en la meta, a la que 
cada uno de los cursos seleccionados para formar parte de cada eje contribuye con el logro de aprendizajes, los que a su vez se convierten en subcompetencias de esa competencia/meta finales.

Así, por ejemplo, en el eje de indagación cada uno de los cuatro cursos que lo componen contribuye con la instalación de aprendizajes, los que sumados, finalmente, darían forma a las competencias a las que alimenta este eje (en resumen, que los estudiantes manejen diversas estrategias de investigación y la capacidad de indagación reflexiva).

De esta forma, el diseño del SME se preocupó de evaluar en cada uno de los cursos de este eje su contribución específica. Para esto, los asesores expertos aconsejaron utilizar como herramienta idealmente una de las evaluaciones ya existentes en los cursos, de manera de no sobrecargarlos de nuevas tareas. A partir de esto, se escogió para cada uno una evaluación (típicamente el examen final) donde se mediría la contribución del curso a las competencias del perfil a las que se asociaba. También, fue necesario construir rúbricas de evaluación que consignaran diferentes niveles de logro para cada una de las dimensiones a evaluar. Como resultado, cada uno de los cursos del eje de indagación tiene, entonces, una evaluación llamada evaluación clave (EC) que alimenta el SME entregando evidencia del logro de las subcompetencias (aprendizajes) con que ese curso contribuye para el logro de las competencias del perfil a las que se asocia por el eje de pertenencia. Asimismo, cada EC entrega datos de los diferentes niveles de logro para cada una de las dimensiones evaluadas en sus rúbricas, y que en conjunto dan forma a las subcompetencias con las que ese curso contribuye al SME.

Esta lógica se aplicó a cada uno de los cinco ejes que forman parte del SME. La lógica de diseño se grafica en la figura siguiente (Figura 4). 
Figura 4: Lógica de diseño del SME

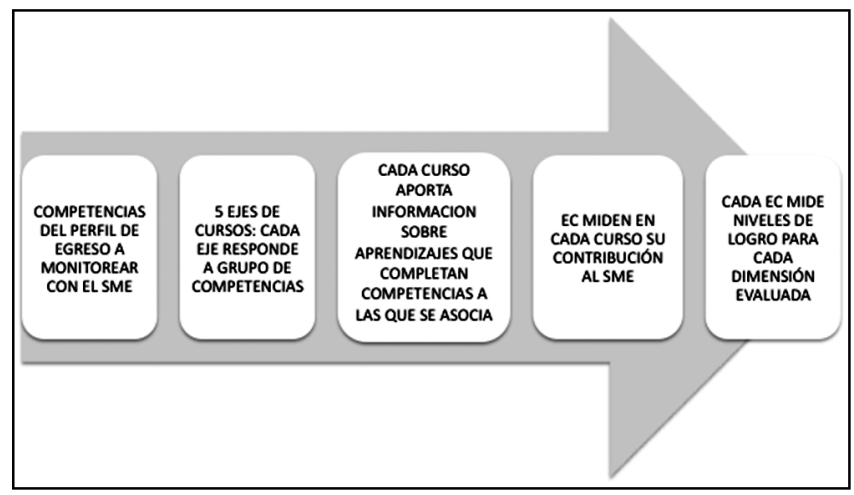

Como resultado de lo anterior, cada EC entrega evidencia (para cada estudiante) acerca de los niveles de logro en las dimensiones medidas. Asimismo, el resultado de las rúbricas informa acerca del logro de las subcompetencias que, a su vez, tributan a las competencias del perfil. Esto, para cada una de las EC de cada curso, perteneciente a cada eje del SME. Pero no todas las EC tienen la misma lógica por medio de los distintos ejes. La mayoría de ellas mide distintos elementos de las competencias a las que tributan, en tanto que otras miden lo mismo mediante el eje, entregando así la posibilidad de observar una ganancia a medida que se avanza.

Así por ejemplo, en el eje de indagación, el primer curso del eje demuestra en su EC el logro de distintas dimensiones evaluadas para conocer si es que los estudiantes adquirieron la capacidad de generar una pregunta de investigación y responderla por medio de la literatura. Para el segundo curso se hizo el mismo ejercicio respecto de la capacidad de aplicar lo anterior e indagar respecto de temas que se enfrentan cotidianamente en las escuelas; y, en los dos últimos cursos, las EC se dirigen a mostrar el logro de las capacidades de generar propuestas de investigación y de ejecutarlas. Cada uno de estos cursos tiene, entonces, diferentes EC y sus rúbricas de corrección.

Por su parte, en el eje de habilidades básicas: alfabetización académica, las EC de los cursos que forman parte del eje son muy similares, ya que todas se dirigen a evaluar la capacidad de los estudiantes de escribir un ensayo académico. Por lo mismo, las 
rúbricas son idénticas y se espera que, a medida que se avance en el eje, el nivel de logro en las EC (y las rúbricas) sea cada vez mayor. Así, se espera que esta lógica de evaluación permita recabar evidencia de ganancias de aprendizaje mientras los estudiantes avanzan en los cursos del eje.

El resto de los ejes no ejemplificados en esta ocasión trabajan con lógicas similares. Toda la información que arroja este sistema se recoge en una plataforma computacional, donde se almacenan las EC para cada uno de los estudiantes, las rúbricas de cada una de ellas y todo el historial de logro para cada uno de los ejes del SME. Cada estudiante tiene acceso a su propia información, cada profesor a los cursos que enseña que son parte del SME, las/los directoras/es a los cursos de su carrera y el secretario académico a la información completa.

El SME se puso en funcionamiento en 2011 para la generación que ingresó ese año, por esto solo una primera etapa de la información ha sido recolectada en la actualidad. Los usos posibles de esta evidencia se discutirán en el apartado siguiente.

\subsection{Uso de la información}

Este sistema pondrá a disposición de la Facultad de Educación de la UDP información que servirá de evidencia para la toma de decisiones de ajuste tanto a nivel de la docencia de cada curso involucrado como de los programas, o de las mallas curriculares de las carreras que participan. Asimismo, mostrará resultados oportunos respecto del logro de las competencias contenidas en el perfil de egreso o, en otras palabras, permitirá contar con datos concretos acerca de la calidad de la FID que se imparte en la Facultad de Educación de la UDP. Lo anterior se aprecia en la Figura 5. 
Figura 5: Uso de la información del SME

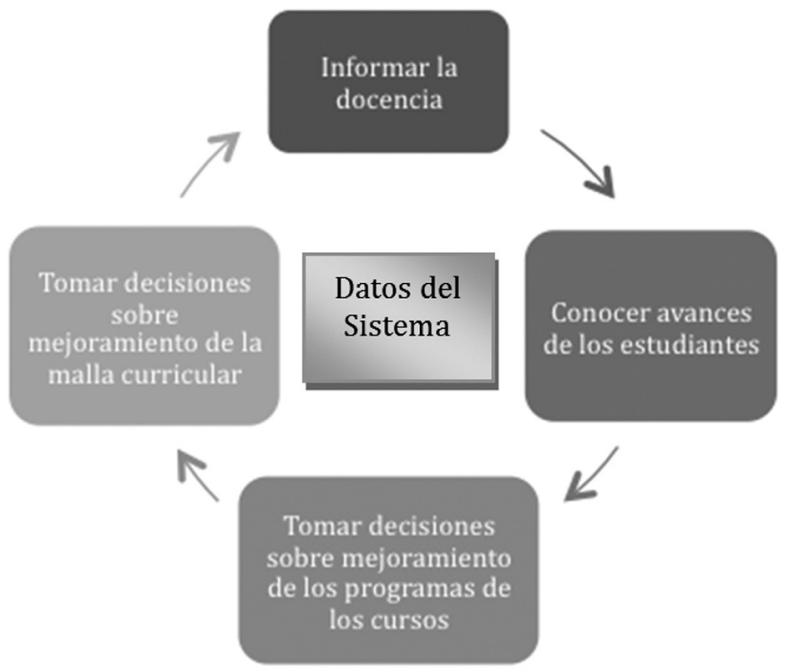

El propósito del SME es brindar la oportunidad de promover una formación de profesores basada en evidencias. Así, se espera que en el futuro los datos que el sistema arroje permitan -al evaluar el logro de los aprendizajes clave por parte de los estudiantes- revisar las prácticas docentes en el aula, los programas de los cursos y el monitoreo y seguimiento del currículo.

Es importante recordar que el SME incluye mediciones no solo al ingreso y egreso de las carreras -como la gran mayoría de los sistemas en uso en otras universidades-, sino también un seguimiento a lo largo de toda la formación universitaria. Esto último permitirá una reacción oportuna cada vez que se detecte que no se están logrando los objetivos que la formación impartida persigue.

Para ejemplificar, un uso más concreto de la evidencia permitirá, desde la misma plataforma que aloja los datos, observar el nivel de logro de un curso en una de las EC (ver Figura 6). 
Figura 6: Ejemplo de niveles de logro de un curso en su EC

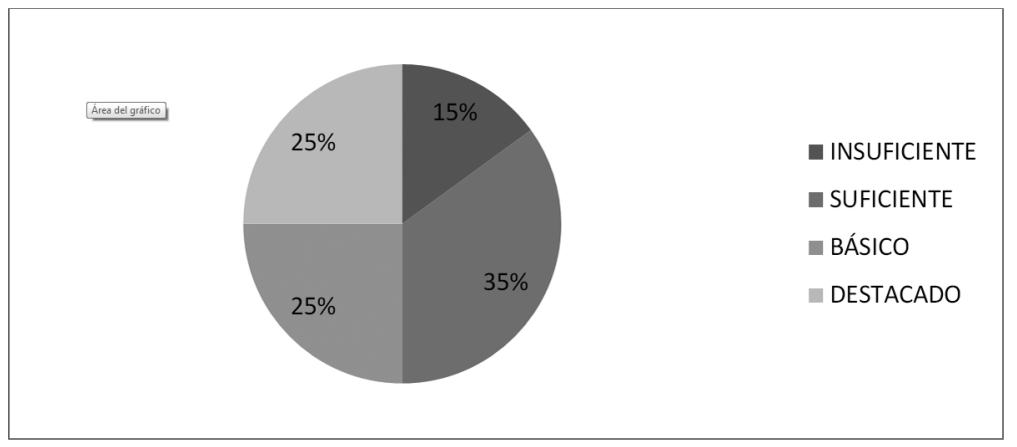

También, los niveles de logro en uno de los ejes para una generación de estudiantes, o bien, el seguimiento de los datos para un estudiante específico.

Los mismos docentes podrán también hacer un estudio en profundidad de los logros en la EC de su curso, observando su rendimiento en cada una de las dimensiones evaluadas con la rúbrica. Esta información podrá iluminar qué aprendizajes fueron los más logrados y cuáles los más débiles, lo que puede tener repercusiones en la posibilidad de instalar acciones remediales futuras, en la manera de hacer la docencia o incluso en la potencial modificación del programa de un curso. En el gráfico siguiente se puede observar un ejemplo de cómo se puede apreciar la diferencia de logro en las distintas dimensiones evaluadas en una EC. En este caso se muestran los resultados de la rúbrica de una de las EC del eje de creencias, en una escala de 0 a 3 puntos (ver Figura 7).

Figura 7: Ejemplo de la información que arroja la aplicación de la rúbrica de una EC (escala de 0 a 3)

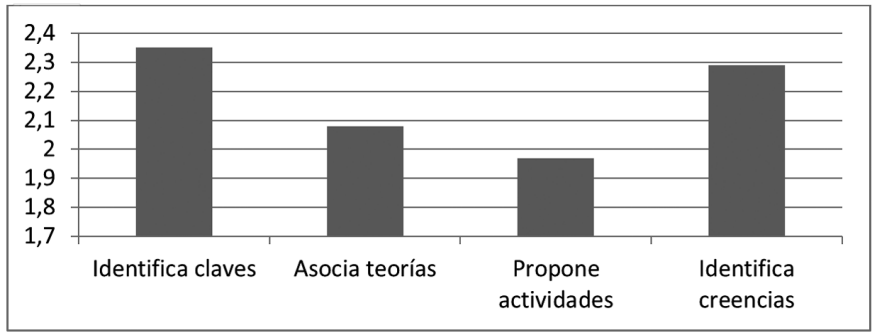


Pero no solo la Facultad y sus profesores podrán monitorear los logros de los aprendizajes, sino que también los mismos estudiantes podrán hacer un seguimiento cercano de su propio proceso, al tener acceso a todos los datos de su recorrido por los cinco ejes y cada una de las EC.

Otro potencial uso de este sistema es que el estudiante de Pedagogía pueda experimentar en sí mismo un modelo de enseñanza donde el qué y cuánto haya aprendido modifica o refuerza el qué y cómo se le enseña, lo que a futuro puede modificar de manera permanente el enfoque de su propia práctica docente en su etapa profesional.

\section{Discusión}

Un sistema de monitoreo como el descrito tiene múltiples usos, potencialidades y también desafíos por enfrentar. Este esfuerzo tiene como meta principal la creación de una cultura de formación de profesores basada en evidencias. Los datos que el SME recoja se convertirán en un insumo clave para evaluar si las metas del currículo de los programas de Pedagogía se han cumplido o no, constituyendo también una importante señal de la calidad de los programas de Pedagogía que la UDP imparte.

Contar con esta evidencia plantea el desafío permanente de revisar acuciosamente los datos de logros, haciéndose cargo de tomar las medidas necesarias para asegurar la instalación de los aprendizajes clave en el mayor número de estudiantes y, de paso, elevar la calidad de la enseñanza que se entrega.

El SME tiene en adelante importantes desafíos, como el perfeccionamiento constante de las EC y sus rúbricas, la revisión de la progresión de los aprendizajes evaluados a lo largo de los ejes, la ampliación a otros saberes que quedan pendientes, la definición de una política de uso de los datos recolectados y la instalación de una cultura de evaluación entre todos los docentes. Todas estas son tareas que se tendrán que abordar año a año para que efectivamente se pueda alcanzar el efecto deseado de elevar la calidad de la enseñanza que 
se entrega. Pero aunque se ha mencionado la necesidad de ajustes y mejoramiento del modelo y sus instrumentos, el solo diseño e implementación de un sistema como este podría marcar una diferencia sustantiva en la calidad de la formación inicial docente, al detectar a tiempo áreas en las que no se están logrando los aprendizajes esperados.

En el primer apartado del presente escrito ya se discutió la importancia que tiene el contar con datos acerca de la eficacia de los programas de FID para asegurar los mejores profesores. Como se ha definido en la Facultad de Educación de la UDP, las carreras de Educación necesitan instalar mecanismos para evaluar el aprendizaje de sus estudiantes a lo largo de la misma, de modo que puedan entregar información a los formadores de profesores respecto del progreso de sus estudiantes en distintos momentos de la carrera. Lo anterior hará posible reaccionar ante debilidades en su aprendizaje, revisando los contenidos y las metodologías de enseñanza para apoyar a los estudiantes antes de su egreso. Esta propuesta se hace aún más urgente en el escenario en que la prueba INICIA se convierta en un examen con repercusiones en el empleo de quienes no logren aprobarlo con niveles aceptables. Esto último impone una gran responsabilidad a las instituciones formadoras respecto de los estudiantes a quienes educa y a sus familias.

Esto nos señala, entre otras cosas, la necesidad de un cuerpo de investigación nacional que pueda informar respecto de qué constituye una formación inicial docente de calidad, qué tipo de formación entregan nuestras universidades en la actualidad y cómo es posible monitorear y evaluar la calidad de dicha formación. De esta forma, examinar los aprendizajes que logran los estudiantes de Pedagogía durante su formación resulta crucial para asegurar la calidad de los profesores que se desempeñen a las escuelas chilenas.

\section{Referencias bibliográficas}

Ávalos, B., (2002) Profesores para Chile. Historia de un proyecto. Santiago, Ministerio de Educación.

Ávalos, B., Cavada, P., Pardo, M. y C. Sotomayor, (2010) «La profesión docente: temas y discusiones en la literatura internacional», en Estudios Pedagógicos, 36, n 1, pp. 235-263. 
Barber, M. y M. Mourshed, (2008) «Cómo hicieron los sistemas educativos con mejor desempeño para alcanzar sus objetivos», en PREAL Documentos, $\mathrm{N}^{\circ} 41$.

Ben-Peretz, M., (2011) «Teacher knowledge: What is it? How do we uncover it? What are its implications for schooling?», en Teaching and Teacher Education, 27, enero , pp. 3-9.

Berliner, D., (1986) «In pursuit of the Expert Pedagogue», en Educational Researcher, 15, agosto-septiembre, pp. 5-13.

Centro de Microdatos, Departamento de Economía Universidad de Chile, (2008) «Estudio sobre causas de la deserción universitaria» [En línea] Disponible en http://www.oei.es/pdf2/causas-desercion-universitariachile.pdf [Recuperado el 21 de abril de 2013]

Darling-Hammond, L., (2006) Power Teacher Education. Lessons from Exemplary Programs. San Francisco, Jossley Bass.

Darling-Hammond, L., (2012) Educar con calidad y equidad. Los dilemas del siglo XX. Santiago, Centro de Innovación en Educación de Fundación Chile.

Darling-Hammond., L. y J. Bransford, (2005) Preparing Teachers for a Changing World. What should Teachers should learn and be able to do. San Francisco, Jossey-Bass.

Ingvarson, L.; Elliott, A.; Kleinhenz, E. y P. McKenzie, (2006) Teacher education accreditation: a review of national and international trends and practices. ACER, Teaching Australia [En línea] Disponible en http://research.acer. edu.au/cgi/viewcontent.cgi?article=1000\&context=teacher_education [Recuperado el 21 de abril de 2013]

Ingvarson, L.; Schwille, J.; Rowley, G.; Tatto, M. T.; Senk, S. y R. Peck, (2011) National Policies and Regulatory Arrangements for the Mathematics Preparation of Future Teachers in Sixteen Countries. Amsterdam, International Association for the Evaluation of Educational Achievement (IEA).

Larrondo, T., (2001) Test de habilidades básicas para estudiantes de Pedagogía. Proceso de validación de las pruebas de habilidades en lenguaje y matemáticas. Valparaíso, Universidad de Playa Ancha.

Larrondo, T.; Lara, M.; Figueroa, C.; Rojas, M. y Caro, A. (2007) Desarrollo de «Habilidades básicas en lenguaje y matemáticas en egresados de Pedagogía. Un estudio comparativo», en Revista Calidad en la Educación, 27, diciembre, pp. 150-176.

Latorre, M.; Aravena, P.; Milos, P. y M. García, (2010) «Competencias habilitantes: un aporte para el reforzamiento de las trayectorias 
formativas universitarias», en Revista Calidad en la Educación, 33, diciembre, pp. 275-301.

Levine, A., (2006) Educating School Teachers. Washington DC., The Education Schools Project.

NCATE. National Council for Accreditation of Teacher Education (2008) What makes a teacher effective? [En línea] Disponible en http:// www.ncate.org/public/researchreports/teacherpreparationresearch/ whatmakesateachereffective/tabid/361/default.aspx [Recuperado el 21 de abril de 2013]

Oliva, I.; Díaz, N.; Larrosa, P.; Contreras, P. y C. Miranda, C., (2010) «Dimensiones de fractura cognitiva en formación inicial docente en Chile: un estudio de casos en tres contextos formativos», en Estudios Pedagógicos, 36, n 1, pp. 177-189. doi: 10.4067/S071807052010000100010.

Rothstein, R.; Jacobsen, R. y T. Wilder, (2009) «From Accreditation to Accountability», en Phi Delta Kappan 90, n 9, mayo, pp. 624-630.

Ruffinelli, A. y A. Guerrero, (2009) «Círculo de segmentación del sistema educativo chileno: destino laboral de egresados de Pedagogía en Educación Básica», en Revista Calidad en la Educación, 31, diciembre, pp. 20-44.

Shulman, L., (1987) «Knowledge and Teaching: Foundations of the New Reform», en Harvard Educational Review, 57, n 1, febrero, pp. 1-21.

Stuhlman, M. y R. Pianta, (2009) «Profiles of Educational Quality in First Grade», en The Elementary School Journal, 109, n 4, pp. 323-342. doi:10.1086/593936.

Velásquez, M.; Peronard, M.; Alonzo, T.; Ibáñez, R. y J. Órdenes, (2006) Guiones metodológicos para desarrollar estrategias de comprensión y producción de textos escritos. Valparaíso, Ediciones Universitarias de Valparaíso.

Wang, A. H.; Coleman, A. B.; Coley, R. J. y R. P. Phelps, (2003) Preparing Teachers around the World. Princeton, Educational Testing Service.

Recibido: 22/04/2013

Aceptado: 21/11/2013 